The work of Chamanlal and Venkataraman ${ }^{4}$ was further described by G. R. M. Garratt who, during a recent visit to New Delhi, was given an opportunity of listening to the meteoric whistles, which were first reported by the Indian workers when listening to the short-wave transmitters of All India Radio. The whistles normally lasted about $2-3$ sec., fell rapidly in pitch to zero or sometimes died away before reaching zero, and only in rare cases did the whistle reappear as an ascending note after passing through zero. Chamanlal and Venkataraman considered that these characteristics could be explained on the assumption that they were due to interference between the directly received ground-wave from the transmitter and the reflected waves which have undergone a Doppler change of frequency owing to the movement of the reflecting surface. The velocities deduced were similar to those of meteors, and the suggestion that the reflexions were associated with meteors was confirmed by direct correlation between the occurrence of a whistle and the arrival of a visible meteor. Mr. Garratt outlined the conditions for satisfactory reception of the meteoric whistles; namely, a high-power transmitter radiating an unmodulated signal at a frequency of the order of 5-15 Mc./s., and a highly sensitive receiver located within the skip distance in a position such that a very weak ground-wave is received from the transmitter. The whistle effects can be heard on almost any night of the year, the greatest number being observed about 4 a.m.

Finally, the recent investigations in the United States by J. A. Pierce ${ }^{9}$ were outlined by K. R. Eldridge. During the Giacobinid shower of October 10,1946 , about 3,500 Doppler whistles were recorded. Pierce considered that the Doppler effect is produced by an 'ionic bow-wave', the pitch being a function of both the velocity of the meteor and the rate of diffusion of the electrons. The pitch is presumed to fall from the first, because the growth of the cloud of ions is most rapid at its initial height, and goes to zero when recombination prevents further expansion of the boundary.

Correlations between the whistles and visually observed meteors were obtained when weather conditions permitted. In the case of two whistles, which were so loud that the visual watchers out-ofdoors $40 \mathrm{ft}$. away could hear them, it was noted that the whistle was heard within the first quarter of the meteor's course, and the tone fell in pitch throughout the period of the meteor's visibility.

Pierce concluded from the average number of whistles observed that his limit of sensitivity corresponded to a third-magnitude meteor. $\mathrm{He}$ calculated that the average rate of flow of energy in the four hours during which the Giacobinid shower was observed corresponded to a figure of 3 watts per sq. $\mathrm{km}$. By assuming that the energy was expended in a layer $30 \mathrm{~km}$. thick, and that 15 electron volts are required per ion, it was calculated that the ion density would be of the same order as the maximum density of ionization in the normal $E$-layer at the sub-solar point.

J. S. Hex

${ }^{1}$ Skellett, Proc. Inst. Rad. Eng., 20, 1933 (1932).

${ }^{2}$ Schafer and Goodall, Proc. Inst. Rad. Eng., 20, 1941 (1932).

${ }^{3}$ Appleton and Naismith, Nature, 158, 936 (1946).

4 Chamanlal and Venkataraman, Electrotechnics, 14, 28 (1941).

${ }^{5}$ Hey and Stewart, Nature, 158, 481 (1946).

' Pierce, Proc. Inst. Rad. Eng., 26, 892 (1938).

${ }^{7} \mathrm{Hey}$, Parsons and Stewart, Mon. Not. Roy. Astro. Soc., in the press.

8. See Nature, 159, 119 (1947).

${ }^{9}$ Pierce, Phys. Rev., 71, 88 (1947).

\section{METEORS, COMETS AND METEORIC IONIZATION}

$\mathrm{O}^{\mathrm{N}}$ March 19 and 20 a conference on meteors, comets and meteoric ionization was held in the University of Manchester Physical Laboratories under the auspices of the Physical Society. The chairman, Prof. P. M. S. Blackett, in opening the conference, remarked that this was probably the first conference of its kind to be held. A widespread interest in meteors and comets is arising because of the new radio methods of detecting the ionization due to meteors in the atmosphere. Results of importance to astronomers and physicists are now being obtained; and it was hoped that this joint gathering would be of benefit to both.

\section{Meteors}

In the first lecture, J. P. M. Prentice, director of the Meteor Section of the British Astronomical Association, described the general background of our knowledge of meteors. The following is a brief abstract of his remarks.

(A) Numbers. The major streams exert a dominant influence on the curve of annual variation, and the rate of occurrence of a given stream is a function of the radiant altitude. The general level of meteor activity after eliminating the major streams is rather low, about 4-10 an hour, and shows daily and annual variations due mainly to the concentration of radiants around the apex of the earth's path. A particular observer sees only a small fraction of the fainter meteors appearing in his zone of vision, so that although the observed numbers fall off after magnitude $4 \cdot 5$, the real numbers continue to increase by a factor $2 \cdot 3$ per magnitude. Beyond the limits of naked-eye visibility, the number of telescopic meteors increases by the same factor at least down to magnitude 10 and probably far beyond.

(B) Radiants. The divergence of meteors from radiant points is an effect of perspective, showing that the motions of those meteors are parallel and that they form members of one stream. These radiants are apparent radiants, due to the combined orbital velocities of the meteors and the earth. Some twelve major streams are known, of which eight appear annually; in addition, there are many minor streams of varying degrees of tenuousness, and some meteors pursuing individual paths in space properly called 'sporadic'. A very few streams are known from the similarity of their orbital elements to be associated with comets, although the proof is only complete for the three instances (possibly four) in which the periods have been determined. The majority of radiants are not points but large areas of radiation some $10^{\circ}-15^{\circ}$ in diameter.

(C) Paths in the Atmosphere. The meteor zone lies generally at an altitude between $125 \mathrm{~km}$. and $70 \mathrm{~km}$., though exact limits cannot be assigned. The height of appearance and disappearance is determined primarily by the entrance velocity, although the beginning height is also correlated with the zenithal magnitude. This difference in height is generally a function of zenithal magnitude only, being sensibly independent of velocity and of radiant altitude except for very low radiant altitudes. The heights are determined by simultaneous observations, which also yield the path-length $P$ and the duration of flight $t$, and hence the obsirved velocity $P / t$. There is some observational evidence of a general decelera- 
tion due to atmospheric resistance, but this is not universally accepted.

(D) Velocities. These may be determined by either direct or indirect methods. The principal direct methods are (a) by simultaneous observations as above, or $(b)$ by observing periodic recurrences of a stream. The indirect methods are (c) from the daily and annual variation in numbers (the method of von Niess $^{1}$ and Hoffmeister ${ }^{2}$, and $(d)$ from the observed heights, which give more reliable values than $P / t$. The only really accurate method is the observation of periodie recurrences, as an error of only $0.5 \mathrm{~km} . / \mathrm{sec}$. usually makes a considerable difference to the orbit; but these recurrences are very difficult to establish. The method of von Niessl and Hoffmeister has led to the belief in hyperbolic velocities as a normal occurrence; but the method is an indirect one and at variance with direct observations and is not considered valid.

\section{Comets and their Relationship with Meteors}

The second lecture was given by Dr. J. G. Porter, the director of the Computing Section of the British Astronomical Association, who spoke on the relation. ship between comets and meteors. Both classes of body are now known to be members of the solar system, travelling around the sun in elliptical orbits; those few cases of hyperbolic orbits which can be substantiated are known to be caused by perturbations of elliptical orbits by the major planets. The head of a comet must possess mass, since it obeys the law of gravitation, but that mass must be very small. All the evidence supports the view that the head consists of solid particles composed of meteoric material. But whereas in a meteor stream the individual particles must be many miles apart (10-20 miles even in the extreme case of the great Leonid shower of 1835), in the head of a comet the distances may be measured in yards. Some force other than gravity is apparently needed, however, to account for the remarkable coherence of the head, which in many comets has undergone enormous disturbances without disruption.

After discussing the effect of planetary perturbations on a meteor stream, Dr. Porter expressed the view that there is no sound evidence that meteor streams are the remains of disrupted comets. The one case which is always quoted-that of Biela's comet-carries very little weight when examined closely. The comet divided into two parts in 1846apparently without cause-was seen once more in 1852 , and then vanished. But in 1872, when the earth crossed the orbit of the comet, a magnificent display of meteors was seen. The meteors could not have been the remains of the head of the comet, for the place where the earth crossed the orbit was far from the computed position of the comet itself. Perturbations by the major planets certainly could not have caused sufficient disturbance in the intervening twenty years to have spread the meteoric matter out over the orbit. All that can be said is that the meteor shower travelled in the same orbit as the comet, and this applies equally to other well-known showers. The Bielids had given good showers before, while the great showers of Lyrids, Leonids and Perseids can all be traced back in history long before the associated comets were discovered.

Actually there are very few accordances between meteor showers and comets-only six of the betterknown showers give satisfactory agreement with their associated comets. Yet there are many minor showers for which the parent comet is unknown, and there are many comets which come close enough to the earth to give a shower, but do not do so.

We are forced to conclude that there is no direct evidence of the disruption of comets to form meteor streams. All that can justly be claimed is that there are some cases known in which a meteor stream follows the same orbit as that of a comet, and it seems plausible to suggest that comets and meteors have a common origin.

\section{Ionization Due to Meteors in the Atmosphere}

In the first lecture of the afternoon session, Dr. A. C. B. Lovell gave a survey of the known effects of meteoric ionization in the earth's atmosphere. Evidence that the ionization in the $E$-region of the ionosphere does not decrease in a regular manner during the night but is subject to abnormal increases was obtained in the early days of the ionosphere work by Heising ${ }^{3}$ in the United States, and by Eckersley ${ }^{4}$, Appleton $^{5}$ and their co-workers in Great Britain. Subsequent work tended to divide the abnormalities into two classes : (a) long-duration increases of ionization lasting for minutes or hours, found on frequencies just above the normal critical frequencies (abnormal or sporadic $E)$; (b) short-duration bursts of ionization lasting for a fraction of a second or a few seconds, which could also be observed on very much higher frequencies. Both effects occurred by day and night throughout the year. As a result of a great deal of work since 1930 by Appleton, Naismith, Rateliffe and others in Great Britain, and by workers in India and the United States ${ }^{6}$, it now seems certain that the long-duration abnormal $E$ is associated with two separate effects: (1) the incidence of solar corpuscles, associated with magnetic disturbances and hence most pronounced in polar regions ; $(2)$ the ionization due to meteors, which constitute the main abnormal effect in temperate latitudes. With regard to $(b)$, Skellett ${ }^{7}$ and Schafer and Goodall ${ }^{8}$ showed, by a combined visual and radio watch during the Leonid shower of 1932, that the short-duration abnormalities were caused by the entry of meteors into the atmosphere.

After describing subsequent work on the shortduration ionization bursts by Eckersley ${ }^{9}$, Appleton and Naismith ${ }^{10}$, Dr. Lovell gave a short account of contemporary studies of the effect using frequencies in the 60-70 Mc./s. region, which are high enough to be clear of the confusing effects of the longer duration abnormal $E$. Hey and Stewart have investigated many of the properties of the radio echoes from meteors and have succeeded in determining radiants $^{11}$ and velocities ${ }^{12}$. In the determination of velocities, these workers were able to photograph a faint fast-moving echo prior to the formation of the main radio echo. The analysis of twenty-two such photographs during the Giacobinid shower of October 1946 gave a velocity of $22.9 \mathrm{~km}$. $/ \mathrm{sec}$., in close agreement with the value of $23.7 \mathrm{~km}$. $/ \mathrm{sec}$. expected from theoretical considerations. The lecture concluded with a short account of the work of the Manchester group, who have explored the major streams by using a narrow beam aerial which can be directed to any point in space. For the meteors in the Giacobinid, Geminid (1946) and Quadrantid (1947) showers the intensity of ionization giving rise to the radio echoes has been calculated ${ }^{23}$. The distribution in number of these intensities obeys an inverse power law with an exponent close to unity. On Herlofson's theory of meteor ionization (see later) this also gives the distribution of meteor masses in the strearn- 
a result in agreement with visual observations that the mass of meteoric material in each magnitude is the same. These measurements also show that a meteor which is just visible to the naked eye produces about $10^{10}$ electrons $/ \mathrm{cm}$. path a result which is again in good agreement with Herlofson's theory ${ }^{14}$. Dr. Lovell ended by showing a ciné film of the radio echoes during the maximum of the Giacobinid shower in October 1946, when the echo-rate reached an enormous peak of 160 per minute for a very short time, compared with the normal no-shower rate of one or two per hour on this apparatus.

\section{Theory of Meteor lonization}

N. Herlofson then gave an account of the theoretical aspects of meteor ionization in the atmosphere. Observations by the Manchester group, referred to above, show that the echoes recorded by their apparatus, at 4 metres wave-length, are due to scattering from columns of electrons with densities of $10^{9}-10^{11}$ electrons per $\mathrm{cm}$. length. These values are in reasonable agreement with quantum-mechanical estimates of the ionization produced by meteors visible to the naked eye, or somewhat fainter. The majority of the echoes disappear after less than one second, a great number lasting only a few tenths of a second; but durations of some seconds are not uncommon, and durations up to 100 seconds have been recorded.

So far, all attempts to find a correlation between duration and height in the atmosphere have been unsuccessful. Another remarkable feature is the occurrence of strong and rapid fluctuations in the intensity of a number of the longer echoes.

A study of the scattering from electrons with a radial Gaussian density-distribution suggests that diffusion is important for the rate of decay of the echoes. Electrostatic forces prevent a separation of the electrons and the positive ions, and the effective diffusion coefficient for the ionized matter will be a weighted mean of the diffusion coefficients for electrons and for positive ions. In a direction normal to the magnetic field of the earth, the diffusion of the electrons will be hampered by the electromagnetic force, while the electromagnetic force does not affect the diffusion in a direction parallel to the magnetic field lines. The resulting elliptical distribution of electrons around the track of the meteor will present its broad side to the radio station in the regions of the sky $90^{\circ}$ away from the magnetic zenith, and its sharp edge if it appears close to the magnetic zenith. Therefore, the duration of the echoes will depend both on height in the atmosphere and on the angular distance from the magnetic zenith to the reflexion point on the meteor traii. In Northern Europe, a belt near the northern horizon will be favourable for echoes of long duration, and some observational evidence already exists in support of this conclusion ${ }^{15}$.

The wind systems revealed by visual observations of the rare luminous trails, lasting for half an hour or more ${ }^{16}$, show that meteor trails can be expected to deviate from a straight line by several metres even after one second. Such deformations can produce a sudden increase of the echo if part of the trail is brought to coincide with one of the spherical wave surfaces. Further deformations may lead to several reflexion points along the trail, and the observed oscillations in echo intensity can be explained by the varying phase difference in the radiation scattered from the various reflexion points.
Since only deformations along the radius from the radio stations are effective, and winds are mainly horizontal, oscillations will preferably occur in echoes observed at low elevations. Combining this conclusion with the expectation of long-duration echoes near the northern horizon, it is possible to understand that long durations are frequently accompanied by fluctuations in intensity at stations in the British Isles.

Observations in equatorial latitudes, where the magnetic field lines are nearly horizontal, can provide a decisive test for these suggestions. We should expect to find echoes of long duration decaying steadily near zenith; echoes of long duration showing fluctuations near the eastern and western horizon, and echoes of shorter duration near the northern and southern horizon.

On March 20, the conference was continued informally at the Jodrell Bank Experimental Station of the University of Manchester, where the apparatus in use by the Manchester group of workers was inspected.

A. C. B. LOVELi

' von Niessl, Astr. Nach., 93, 209 (1878).

"Hoffmeister, "Das Interstellare System der Kleinkorper" (1936).

${ }^{3}$ Heising, Proc. Inst. Rad. Eng., 16, 75 (1928).

' Eckersley, J. Inst. Elect. Eng., 67, 992 (1929).

'Appleton, Proc. Roy. Soc., A, 128, 542 (1930).

- See particularly, Appleton and Naismith, Proc. Roy. Soc., A, 137 36 (1932); Proc. Phys. Soc., 45, 389 (1933); Phil. Trans., A 236, 191 (1937). Appleton, Naismith and Ingram, Proc. Phys. Soc., 51, 81 (1929). Appleton and Naismith, Proc. Phys. Soc. 52, 402 (1940); also in course of publication. Ratcliffe and White, Proc. Phys. Soc., 46, 107 (1934). Best, Farmer and Ratcliffe, Proc. Roy. Soc., A, 164, 93 (1938). Mitra. Syam and Ghose, Nature, 133, 533 (1934). Bhar, Ind. J. Phys., i1, 109 (1937).

7 Skellett, Proc. Inst. Rad. Eng., 23, 132 (1935).

- Schafer and Goodall, Proc. Inst. Rad. Eng., 20, 1131 (1932); 20, 1941 (1932).

- Eckersley, J. Inst. Elect. Eng., 71, 405 (1932); 86, 548 (1940). Nature, 140, 846 (1937). Éckersley and Farmer, Proc. Roy. Soc., A, 184, 196 (1945).

${ }^{10}$ Appleton, Naismith and Ingram, Phil. Trans., A, 236, 191 (1937). Appleton and Piddington, Proc. Roy. Soc., A, 164, 467 (1938). ${ }^{11} \mathrm{Hey}$ and Stewart, Nature, 155, 481 (1946).

${ }^{12} \mathrm{Hey}$, Stewart and Parsons, Mon. Not. Roy. Astro. Soc., in the press. ${ }^{13}$ Lovell, Banwell and Clegg, Mon. Not. Roy. Astro. Soc., in the press. ${ }^{14}$ Prentice, Lovell and Banwell, Mon. Not. Roy. Astro. Soc., in the press.

${ }^{15}$ Lovell and co-workers, unpublished.

${ }^{16}$ For example, Störmer, C., Astrophys. Nor., Oslo, III, No. 5 (1939).

\section{NUTRITION AND SYMBIOSIS*}

\section{By Dr. E. C. OWEN}

Hannah Dairy Research Institute, Kirkhill, Ayr

TUTRITION may be taken to include all those processes by which chemical substances essential to the life of a given species, be it plant or animal, become available for anabolism. Thus in plants, nutrition includes photosynthesis and root absorption, as well as the processes of digestion which occur in animals and insectivorous plants. The term 'symbiosis' in its original and widest sense signifies any phenomenon, from a balanced parasitism to complete mutualism, in which each partner gains nutritional benefit from the presence of the other. Such diverse phenomena as the harbouring of specific fauna and flora in the gut of insects and vertebrates and the occurrence of algæ in the cells of Hydra viridis are, in the widest sense of the term, symbiotic.

Symbiosis is a phenomenon of very general occurrence in both the plant and the animal realms.

* Paper read before the Society for Experimental Biology on September 25, 1946. 